\title{
Default Correlations and Large-Portfolio Credit Analysis*
}

\author{
Jin-Chuan Duan ${ }^{\dagger}$ and Weimin Miao ${ }^{\ddagger}$
}

\section{Appendices}

\section{A: Sparse correlation matrix estimation with missing data}

Consider an incomplete data set $Z$ of size $T \times n$ containing $n$ time series of $T$ observations, where $n \gg T$. Our task is to find an appropriate estimate for the $n \times n$ correlation matrix $\Sigma$ by imposing sparsity, namely, many entries are zeros.

In the high-dimensional setting, the sample correlation matrix is no longer consistent with its population counterpart, and regularization is needed for obtaining an appropriate estimate. We follow Bickel and Levina (2008b), Rothman, et al (2009), and Cai and Liu (2011) to apply elementwise thresholding to the sample correlation matrix to accommodate the sparsity assumption. We choose the thresholding method rather than the lasso-type penalization method for sparsity because the former has computational efficiency, particulary when dealing with matrices of a very large size. It is worth noting that thresholding on correlations functions as a statistical significance filtering when dealing with complete data. With missing data, however, only the pairwise sample correlation matrix is available and different entries naturally face different degrees of freedom. Thus, our approach comprises two steps.

Step 1: (Significance filtering) Compute the pairwise sample correlation matrix, and then set an entry to zero if it is not statistically significant based on the two-tailed $p$-value at $5 \%$. The resulting correlation matrix is denoted by $\tilde{\Sigma}=\left(\tilde{\sigma}_{i j}\right)_{n \times n}$.

\footnotetext{
${ }^{*}$ This paper originated from several discussions with Rowan Douglas and David Simmons of Willis on how the CRI database of Risk Management Institute, National University of Singapore can be intelligently applied to help insurers manage their credit portfolios. This project benefits from RMI-CRI being a member of the Willis Research Network at the time. The authors would like to acknowledge the helpful comments received from the seminar participants at Auckland Technology University, Federal Reserve Board, New York University, Princeton University and Bank of Canada, and also at the 2015 International Conference on Financial Engineering \& Innovation in Chengdu, China.

${ }^{\dagger}$ Duan is with the National University of Singapore (Risk Management Institute, Business School and Department of Economics). E-mail: bizdjc@nus.edu.sg.

${ }^{\ddagger}$ Miao is with the National University of Singapore (Risk Management Institute). This research began when Miao was a Willis Research Fellow. E-mail: rmimw@nus.edu.sg.
} 
Step 2: (Thresholding) Apply thresholding on $\hat{\Sigma}^{(s)}$. Define

$$
\hat{\Sigma}^{(\tau)}:=\left(\hat{\sigma}_{i j}^{(\tau)}\right)_{n \times n} \quad \text { with } \quad \hat{\sigma}_{i j}^{(\tau)}:= \begin{cases}1 & \text { for } i=j \\ s_{\tau}\left(\tilde{\sigma}_{i j}\right) & \text { for } i \neq j\end{cases}
$$

where $s_{\tau}(\cdot)$ is a thresholding function associated with some constant threshold $\tau \in[0,1]$.

The thresholding function typically includes hard-, soft- and SCAD-thresholdings. We adopt the SCAD approach:

$$
s_{\tau}(z)= \begin{cases}\operatorname{sgn}(z)(|z|-\tau)_{+} & \text {for }|z| \leq 2 \tau \\ {[(a-1) z-\operatorname{sgn}(z) a \tau] /(a-2)} & \text { for } 2 \tau<|z| \leq a \tau \\ z & \text { for }|z|>a \tau\end{cases}
$$

where $\operatorname{sgn}(\cdot)$ is the sign function and $x_{+}:=\max \{x, 0\}$. Our implementation sets $a=3.7$ as recommended by Fan and $\mathrm{Li}(2001)$.

Due to missing data, the pairwise sample correlation matrix may not be positive semidefinite. We adopt the method of Fan, et al (2013) to ensure finite-sample positive semidefiniteness. Note that when the threshold $\tau$ is sufficiently close to 1 , the estimator $\hat{\Sigma}^{(\tau)}$ becomes diagonal and thus positive definite. Define $\tau_{\min }:=\min \left\{\tau \geq 0 \mid \lambda_{\min }\left(\hat{\Sigma}^{\left(\tau^{\prime}\right)}\right) \geq 0, \forall \tau^{\prime} \geq \tau\right\}$, where $\lambda_{\text {min }}$ denotes the smallest eigenvalue. The relevant threshold is thus narrowed to the set, $\left[\tau_{\min }, 1\right]$. (Note: We do not need to invert the correlation matrix for our problem, and therefore positive semidefiniteness is sufficient. Otherwise, one may slightly increase $\tau_{\min }$ to obtain positive definiteness.)

Search the threshold $\tau \in\left[\tau_{\min }, 1\right]$ via random subsampling cross-validation. We follow the procedure introduced by Bickel and Levina (2008b) and analysed by Bickel and Levina (2008a). The data matrix $Z$ is randomly split into two subsets - training set $Z_{1}$ and validation set $Z_{2}$. The sizes of $Z_{1}$ and $Z_{2}$ are, respectively, $T_{1} \times n$ and $T_{2} \times n$, where $T_{2}=T / \ln (T)$ and $T_{1}=T-T_{2}$. We repeat the split $K$ times. For the $k$-th split, we denote by $\hat{\Sigma}_{1}^{(\tau, k)}$ the resulting correlation matrix after applying both of the two regularization steps (significance filtering and thresholding) to the training data set, and denote by $\tilde{\Sigma}_{2}^{(k)}$ the resulting correlation matrix after only applying the first regularization step (significance filtering) to the validation data set. We then choose the best threshold $\tau^{*}$ by minimizing a cross-validation objective function over the range that positive semidefiniteness is guaranteed, i.e.,

$$
\tau^{*}=\underset{\tau_{\min } \leq \tau \leq 1}{\arg \min } \frac{1}{K} \sum_{k=1}^{K}\left\|\hat{\Sigma}_{1}^{(\tau, k)}-\tilde{\Sigma}_{2}^{(k)}\right\|_{F}^{2}
$$

where $\|\cdot\|_{F}$ stands for the Frobenius norm. In our implementation, $K$ is set to 10.

\section{B: Algorithms for default-rate and portfolio-loss distributions}


Consider a credit portfolio of $n$ obligors. The algorithms are for computing the distributions for this portfolio's default rates and loss rates over a specified time period. For obligor $i(i=1, \cdots, n)$, let $p_{i}, r_{i}$ and $z_{i}$ denote its $\mathrm{PD}$ over the time period, recovery rate at default, and exposure amount, respectively. We assume random recovery rates with known distributions, and they may be different across different obligors. If recovery rates are constants, the algorithm for portfolio-loss distribution can be significantly simplified. The method for the default-rate distribution is taken from Duan (2010). The algorithm for the portfolio-loss distribution is new, which takes advantage of the default-rate distribution.

The two algorithms described below are conditional on the random PDs generated by the factor model. One needs to repeatedly apply the algorithm to obtain many random copies of the defaultrate (or portfolio-loss) distribution, and then average them to obtain a Monte Carlo estimate of the final distribution.

\section{B.1: Default-rate distribution conditional on randomized PDs}

Denote the default-rate distribution of this portfolio by $Q(k / n), k=0, \cdots, n$. Obviously, $Q(k / n)=P_{n}(k)$, where $P_{i}(k)$ represents the probability of $k$ defaults in this portfolio after first $i$ out of $n$ obligors have been considered. Instead of tacking the default numbers that have negligible probabilities, we truncate $P_{i}(k)$ at some default number, $k^{*}$, beyond which the probability is smaller than a tiny threshold, $\tau$, which is set to $10^{-6}$. The following convolution method follows that of Duan (2010), but is stated in an algorithm style. The convolution algorithm is as follows:

Step 0: Let $P_{0}(0):=1$. Set $k^{*}:=0$ and $i:=1$.

Step 1: Forward the convolution to the next obligor by letting

$$
P_{i}(k):= \begin{cases}P_{i-1}(0)\left(1-p_{i}\right) & \text { for } k=0 \\ P_{i-1}(k)\left(1-p_{i}\right)+P_{i-1}(k-1) p_{i} & \text { for } k=1, \cdots, k^{*} \\ P_{i-1}(k-1) p_{i} & \text { for } k=k^{*}+1\end{cases}
$$

Step 2: Reset the truncated point $k^{*}:=\max _{1 \leq k \leq k^{*}+1}\left\{P_{i}(k) \geq \tau\right\}$. If $i=n$, reset $P_{n}(\cdot)$ by dividing this vector with its own sum to ensure no loss of probability, and let

$$
Q(k / n)= \begin{cases}P_{n}(k) & \text { for } 0 \leq k \leq k^{*} \\ 0 & \text { for } k^{*}<k \leq n\end{cases}
$$

and stop. Otherwise, reset $i:=i+1$, and go to Step 1 .

\section{B.2: Portfolio-loss distribution conditional on randomized PDs}

With the default-rate distribution in place, we still need to deal with the significant complication associated with heterogeneity across obligors in terms of their individual exposures and recovery 
rates. We assume recovery rates are independent across obligors at the time of their defaults. Convolution can be applied to individual loss distributions (combining an obligor's default probability with its loss-given-default distribution) one obligor at a time. Facing a portfolio of many obligors, this approach would be highly inefficient. An alternative is provided in Gordy (2002), which has long been used in the insurance literature via approximating the product of the generating functions for individual obligors' overall loss by an exponential of the sum of the generating functions for individual loss-given-default, assuming that these generating functions for individual loss-givendefault are readily available. This approximation works well when the PDs for the obligors in the portfolio are small, which in our case is unlikely simply because there are a large number of obligors and the aggregation is performed on the randomized PDs.

Our approach described below is novel and efficient in dealing with a large number of obligors. First, we recognize that if the identities of the defaulters are known, their loss-given-default distributions can be easily convoluted. Since many obligors default at the same time has a very low probability, such convolution only needs to be performed on a small set of obligors. However, we do not know who the defaulters out of the total $n$ obligors will be. Exhausting all possible combinations, knowing the likelihood of each combination, and computing the convoluted loss distribution for each combination can be a daunting task. Our novel simulation-convolution method relies on randomizing over the identities of defaulting obligors.

Let $0=c_{0}<c_{1}<\cdots<c_{m-1}<c_{m}=1$ be a partition of the percentage loss domain [0,1] into $m$ equally sized subintervals. Define $I_{0}=\{0\}$ and $I_{j}:=\left(c_{j-1}, c_{j}\right], j=1, \cdots, m$. Denote by $D\left(I_{j}\right)$, $j=0, \cdots, m$, the discretized loss distribution of $n$ obligors that is associated with the partition. For each obligor, we convert its individual loss distribution into one that is measured as a fraction of the total portfolio exposure and based on the partition; that is,

$$
q_{i}\left(I_{j}\right)=\operatorname{Prob}\left(\frac{z_{i}\left(1-r_{i}\right)}{\sum_{i=1}^{n} z_{i}} \in I_{j}\right), \quad i=1, \cdots n, j=0, \cdots, m .
$$

Similar to the algorithm for the default-rate distribution, we determine $k^{*}$ by setting $\tau=10^{-6}$ to truncate the loss distribution function beyond which the chance of occurrence is practically zero. The algorithm is as follows:

Step 0: Obtain the distribution for the number of defaults, i.e., $P_{n}(k), k=0, \cdots, k^{*}$, as in the convolution algorithm for the default-rate distribution.

Step 1: Simulate a set of indices of $k^{*}$ defaulting obligors, say $i_{1}, \cdots, i_{k^{*}}$, one at a time using an appropriate probability for each of the remaining obligors. Specifically, let $A_{k}$ be the set of remaining obligors after sampling out the first $k-1$ defaulting obligors. The probability for sampling $i_{k}$ is $\frac{p_{i} \prod_{\left\{l \in A_{k}, l \neq i\right\}}\left(1-p_{l}\right)}{\sum_{j \in A_{k}} p_{j} \prod_{\left\{l \in A_{k}, l \neq j\right\}}\left(1-p_{l}\right)}$ for $i \in A_{k}$.

Step 2: Use convolution to calculate a sequence of loss distributions, each conditional on the simulated $k$ defaulting obligors, $k=1, \cdots, k^{*}$. The randomized loss distribution after completing $k$ 
out of $k^{*}$ obligors is denoted by $\tilde{Q}_{k}\left(I_{j}\right), j=0, \cdots, m$. Apply a threshold $\tau^{\prime}:=\frac{\tau}{\max _{1 \leq k \leq k^{*}}\left\{P_{n}(k)\right\}}$ to truncate each loss distribution. (Note: $\tau^{\prime}$ is used to ensure that each random copy of $\tilde{Q}_{k}(\cdot)$ after multiplying by $P_{n}(k)$ later is effectively truncated by $\tau$. This treatment is used to speed up the algorithm while maintaining numerical accuracy.)

Step 2.0: Let $\tilde{Q}_{0}\left(I_{0}\right):=1$ and $\tilde{Q}_{0}\left(I_{j}\right):=0$ for $j=1, \cdots m$. Set $s_{1}:=0, s_{2}:=0$ and $k:=1$.

Step 2.1: Set $t_{1}:=\min _{0 \leq j \leq m}\left\{q_{i_{k}}\left(I_{j}\right) \geq \tau^{\prime}\right\}$ and $t_{2}:=\max _{0 \leq j \leq m}\left\{q_{i_{k}}\left(I_{j}\right) \geq \tau^{\prime}\right\}$. Set $j_{1}:=$ $s_{1}+t_{1}$ and $j_{2}:=s_{2}+t_{2}$. Let

$$
\tilde{Q}_{k}\left(I_{j}\right)=\left\{\begin{array}{cc}
\tilde{Q}_{k-1}\left(I_{\max \left\{s_{1}, j-t_{2}\right\}}\right) q_{i_{k}}\left(I_{\min \left\{t_{2}, j-s_{1}\right\}}\right)+\cdots & \text { for } j_{1} \leq j \leq j_{2} \\
+\tilde{Q}_{k-1}\left(I_{\min \left\{s_{2}, j-t_{1}\right\}}\right) q_{i_{k}}\left(I_{\max \left\{t_{1}, j-s_{2}\right\}}\right) & \text { for } 0 \leq j<j_{1} \text { and } j_{2}<j \leq m \\
0 &
\end{array}\right.
$$

Step 2.2: If $k=k^{*}$, go to Step 3. Otherwise, set $s_{1}:=\min _{j_{1} \leq j \leq j_{2}}\left\{\tilde{Q}_{k}\left(I_{j}\right) \geq \tau^{\prime}\right\}, s_{2}:=$ $\max _{j_{1} \leq j \leq j_{2}}\left\{\tilde{Q}_{k}\left(I_{j}\right) \geq \tau^{\prime}\right\}$ and $k:=k+1$, and return to Step 2.1.

Step 3: Compute the randomized version of the portfolio-loss distribution by

$$
\tilde{D}\left(I_{j}\right):=\sum_{k=0}^{k^{*}} P_{n}(k) \tilde{Q}_{k}\left(I_{j}\right), \quad j=0, \cdots, m
$$

and then reset $\tilde{D}(\cdot)$ by dividing this vector with its own sum to ensure no loss of probability.

The percentage loss partition needs to be fine enough to achieve good approximation quality, and we set $m=50,000$ in the implementation. Corresponding to each simulation of one-month PDs and POEs, we generate one random copy of $\tilde{D}(\cdot)$. 